HU-EP $01 / 30$

hep-th/0107264

\title{
Type IIB supergravity compactified on a Calabi-Yau manifold with $H$-fluxes
}

\author{
Gianguido Dall'Agata ${ }^{\sharp}$ \\ \# Institut für Physik, Humboldt Universität \\ Invalidenstraße 110, 10115 Berlin, Germany \\ dallagat@physik.hu-berlin.de
}

\begin{abstract}
We discuss the compactification of type $I I B$ supergravity on a Calabi-Yau manifold in the presence of both $R R$ and $N S$ fluxes for the three-form fields. We obtain the classical potential both by direct compactification and by using the techniques of $\mathcal{N}=2$ gauged supergravity in four-dimensions. We briefly discuss the properties of such potential and compare the result with previous derivations.
\end{abstract}




\section{Introduction}

It is a known fact that the compactification of type $I I B$ supergravity on a six-dimensional Calabi-Yau manifold gives rise to an effective four-dimensional theory which is given by $\mathcal{N}=2$ supergravity coupled to $h_{(2,1)}$ vector multiplets and $h_{(1,1)}+1$ hypermultiplets. The addition of non-trivial fluxes for the Neveu-Schwarz and Ramond three forms make the universal hypermultiplet charged under the axionic shift symmetries of the moduli-space. As a consequence, the scalar sector develops a non-trivial potential whose critical points determine new vacua of the theory, possibly breaking supersymmetry to $\mathcal{N}=1$ or $\mathcal{N}=0$.

The phenomenon of partial supersymmetry breaking occurs very rarely for $\mathcal{N}=2$ theories in $D=4$ [1, 2, 3] and therefore it is very interesting to see if there is any chance to obtain it in this framework. Moreover, this scenario could provide a mechanism to construct $\mathcal{N}=1$ gauge theories in the limit of decoupling of gravity.

As one could expect, this kind of theories have already been considered in the past. The general features of the compactifications without fluxes have been discussed in [⿶] whereas the details have been worked-out in [5].

For what concerns the theories in the presence of fluxes, the vacua of the derived potential have been studied in [6], claiming that one can have only $\mathcal{N}=2$ or $\mathcal{N}=0$ supersymmetry preserved. In [7] it is further argued that one can produce $\mathcal{N}=1$ potentials considering non-compact Calabi-Yau manifolds and taking a certain decompactification limit that makes some degrees of freedom non-dynamical. In particular, it was derived the expression of the final $\mathcal{N}=1$ superpotential $W$ in terms of the fluxes, according with the general analysis of [8]. In [9, 10] this same $W$ was related to the four-dimensional quaternionic prepotentials and it was performed the analysis of the supersymmetric vacua of such theory for singular Calabi-Yau manifolds.

In this paper we address once more the problem of the derivation of the four-dimensional theory and the study of its vacua. We will derive the classical potential of the fourdimensional effective theory both by direct compactification and by using the techniques of $\mathcal{N}=2$ gauged supergravity. As expected, the two results agree. This fact, that could seem trivial, was instead a controversial point of previous derivations. In particular, it was known that the potentials derived by Michelson [6] (using the $\mathcal{N}=2$ gauged supergravity techniques) and by Taylor and Vafa [7] (using direct compactification) did not agree. For instance the potential presented in [7] is positive semi-definite, whereas the one in [6] is not. Moreover, as noted in [9], the potential of [7] is also $S L(2, \mathbb{Z})$ invariant and this feature is not present in the one of [6].

We also show that the resulting potential has a run-away behaviour. This implies that no critical points at all are allowed in the classical region of validity of the scalar-manifold variables. In particular, to obtain critical points, the volume of the Calabi-Yau is driven to infinity. We don't discuss here the possibility of using singular manifolds along the lines of [7, 9, 10], which we leave for the future. 
We will also briefly discuss some interesting properties of such potential, with a special attention to the possibility of writing it in the $\mathcal{N}=1$ form presented in [7]. We will show that this cannot be done for the generic case, but that only a truncation of some of the hyper-scalars can lead to such a form. Although this cannot be done retaining an $\mathcal{N}=2$ theory, it could happen that for some consistent reduction to $\mathcal{N}=1$ this possibility appears.

We will also discuss the supersymmetry laws of the Fermi fields, showing that indeed one cannot find $\mathcal{N}=1$ configurations unless the hyperinos are discarded. This leaves open the possibility to obtain a consistent $\mathcal{N}=1$ theory in the limit where some of the moduli are freezed.

\section{Compactification of type $I I B$ supergravity}

Let us start computing what is the effect of turning on a v.e.v. for the three form field strength in type IIB supergravity when we compactify the theory down to four dimensions on a Calabi-Yau manifold.

For the sake of semplicity, we will consider here a manifold with arbitrary $h_{(2,1)}$ but $h_{(1,1)}=1$, which is the minimum required by a Calabi-Yau. This implies that the number of hypermultiplet is fixed to two and that they parametrize the quaternionic manifold given by $G_{2(2)} / S O(4)$ 11].

On the Calabi-Yau manifold $\mathcal{M}$, we take a canonical homology basis for $H_{3}$ given by $A^{\Lambda}, B_{\Lambda}\left(\Lambda=0,1, \ldots, b_{2,1}\right)$ and let $\left(\alpha_{\Lambda}, \beta^{\Lambda}\right)$ be the dual cohomology basis. Completeness of this basis implies that

$$
\int_{A^{\Lambda}} \alpha_{\Pi}=\int_{\mathcal{M}} \alpha_{\Pi} \beta^{\Lambda}=\delta_{\Pi}^{\Lambda}
$$

as well as the converse

$$
\int_{B_{\Pi}} \beta^{\Lambda}=\int_{\mathcal{M}} \beta^{\Lambda} \alpha_{\Pi}=-\delta_{\Pi}^{\Lambda}
$$

Since we are going to discuss the effect of turning on $H$-fluxes, we will give an expectation value to the Ramond and Neveu-Schwarz three-forms as

$$
\left\langle H^{1}\right\rangle=e_{\Lambda}^{1} \beta^{\Lambda}, \quad\left\langle H^{2}\right\rangle=e_{\Lambda}^{2} \beta^{\Lambda}
$$

This, of course, is not the most general form of fluxes one can turn on, but in what follows we will limit the analysis only to electric charges. We choose to impose this restriction, because we want to make a comparison with results that can be obtained from the standard four-dimensional gauged supergravity and therefore the electric charges are the only ones which can receive a correct treatment in such framework 12]. Anyway it is not difficult to believe that the results could be extended to the presence of magnetic charges, as well. It is known that these charges can be obtained by a rotation in the symplectic base used for describing the special Kähler geometry of the vector scalar manifold. 
We expect that the new contributions to the ten-dimensional theory coming from the above fluxes (2.3) are given by new terms or modifications to the kinetic terms of the three and five forms and to the Wess-Zumino term. These terms will produce a fourdimensional scalar potential as well as new interactions between the scalar and vector fields of the theory. In particular they will make charged some of the scalars under two abelian gauge symmetries.

Since we are mainly interested in the final scalar potential, in the following we will consider only the bosonic sector of such theory. This is indeed enough to read directly the result for such potential, as well as to read the quantities needed to identify the four-dimensional gauged theory which reproduces such result.

Our starting point will be the covariant action for the ten-dimensional theory of type $I I B$ supergravity of 13, 14. In this action, the problem of obtaining the self-duality constraint of the $R R$ five-form without compromising Lorentz invariance is solved by using the PST formalism [15]. This implies the addition of a scalar field $a(x)$, singlet under supersymmetry, which is a pure gauge under additional symmetries that the action now exhibits.

Of course, when we compactify this action to four dimensions, this extra field should not contribute and so we just set it to zero, discarding then also all the terms which contain it. Doing this, the relevant part of the starting action is given by 14

$$
\int d^{10} x \sqrt{-g} R+\frac{1}{4} \int F_{5} \star F_{5}+2 \int \bar{F}_{3} \star F_{3}+2 \int \bar{F}_{1} \star F_{1}+\frac{\mathrm{i}}{2} \int(B \bar{H}-\bar{B} H) F_{5}
$$

where the definition of the various forms is

$$
\begin{array}{ll}
F_{1}=\bar{U} d V-V d \bar{U}, & F_{3}=\bar{U} H+V \bar{H} \\
F_{5}=d A_{4}+\mathrm{i}(B \bar{H}-\bar{B} H), & H=d B
\end{array}
$$

and $\star$ denotes the Hodge-duality operation. The $(U, V)$ scalars are the dilaton/axion of the ten dimensional theory parametrizing the $S U(1,1) / U(1)$ manifold. This implies that they must satisfy the constraint $|U|^{2}-|V|^{2}=1$. At the same time they define the $U(1)$ connection $Q=-\frac{\mathrm{i}}{2}(\bar{U} d V-V d \bar{U})$.

From this action we now want to compute the scalar potential of the four-dimensional theory, consequence of the introduction of the (2.3) fluxes.

To do so and also make contact with previous work, we make the following field redefinitions

$$
U=\frac{1}{\sqrt{1-\psi \bar{\psi}}}, \quad V=\frac{\psi}{\sqrt{1-\psi \bar{\psi}}}
$$

with

$$
\psi=\frac{1-\eta}{1+\eta}, \quad \text { and } \quad \eta=\alpha+i \beta, \quad\left(\alpha=e^{-\varphi_{10}}\right)
$$


We can also decompose $H$ in its real and imaginary part $H=H_{1}+\mathrm{i} H_{2}$, such that we can rewrite the kinetic term for the three-forms as

$$
2 \bar{F}_{3} \star F_{3}=\frac{4}{\eta+\bar{\eta}}\left[\left(H_{1}-\mathrm{i} \bar{\eta} H_{2}\right) \star\left(H_{1}+\mathrm{i} \eta H_{2}\right)\right] .
$$

Defining now

$$
e_{\Lambda} \equiv e_{\Lambda}^{1}+\mathrm{i} \eta e_{\Lambda}^{2}
$$

we can compute the potential following the lines of [7]. Indeed, we can write (2.8) as

$$
2(\operatorname{Re} \eta)^{-1} \int \bar{e}_{\Lambda} \beta^{\Lambda} \wedge \star\left(e_{\Sigma} \beta^{\Sigma}\right)
$$

and make use of the duality relations between the Hodge-forms for a Calabi-Yau threefold [16].

If indeed we call $\mathcal{R} \equiv \operatorname{Re} \mathcal{N}, \mathcal{I} \equiv \operatorname{Im} \mathcal{N}$, where $\mathcal{N}_{\Lambda \Sigma}$ is the matrix of the kinetic term for the vector fields in $D=4$, the Hodge duality of the forms reads

$$
\begin{aligned}
\star \alpha_{\Lambda} & =\left(\mathcal{R} \mathcal{I}^{-1}\right)_{\Lambda}{ }^{{ }} \alpha_{\Sigma}-\left(\mathcal{I}+\mathcal{R} \mathcal{I}^{-1} \mathcal{R}\right)_{\Lambda \Sigma} \beta^{\Sigma} \\
\star \beta^{\Lambda} & =\mathcal{I}^{-1 \Lambda \Sigma} \alpha_{\Sigma}-\left(\mathcal{I}^{-1} \mathcal{R}\right)^{\Lambda}{ }_{\Sigma} \beta^{\Sigma}
\end{aligned}
$$

Using (2.11), (2.12) and the relation coming from the special Kähler geometry [4]

$$
U^{\Lambda \Sigma} \equiv f_{i}^{\Lambda} g^{i \bar{\jmath}} f_{\bar{\jmath}}^{\Sigma}=-\frac{1}{2} \mathcal{I}^{-1 \Lambda \Sigma}-\bar{L}^{\Lambda} L^{\Sigma}
$$

it is straightforward to show that (2.10) becomes

$$
\frac{8}{\eta+\bar{\eta}} e_{\Lambda} \bar{e}_{\Sigma}\left(\bar{L}^{\Lambda} L^{\Sigma}+U^{\Lambda \Sigma}\right)
$$

This (besides normalizations) is the same result obtained in [7]. Anyway, we will see in a moment that this is not the final result for the potential of the theory, since, to go in the Einstein frame and obtain the correct normalization for the kinetic terms, we still need to rescale the metric. This additional factor will reveal crucial to obtain an agreement with the gauged supergravity result.

We now show that the above potential (2.14) does not receive further contributions from the other terms in the action and also fix the amount of the rescaling needed to go to the four-dimensional Einstein frame. We will also compute the various kinetic terms for the scalars and vectors and their couplings to determine the form of the four-dimensional supergravity action to which we want to compare our results. To simplify the reading of this technical part, we divide the various sectors of the compactification according to the expansion of the various fields into different harmonic forms. Moreover, we will mainly quote the results, detailing only the derivation of some very important structures.

First we will compute the contributions of the five-form field (through its kinetic term and the Wess-Zumino term) to the kinetic terms of the vectors and the couplings of some 
scalars with the vectors through the $H^{3}$ sector. Then we will analyze the $H^{2}$ and $H^{0}$ sectors computing the kinetic terms of the various four-dimensional scalars. This will fix completely the four-dimensional theory and determine the gauging one needs to perform.

Let us start with the $H^{3}$ sector. The zero modes of the five-form on the Calabi-Yau manifold, now that we have included also the three-form fluxes, are given by

$$
F_{5}=F^{\Lambda} \alpha_{\Lambda}-G_{\Lambda} \beta^{\Lambda}+2 B^{i} e_{\Lambda}^{j} \beta^{\Lambda} \epsilon_{i j}
$$

This implies that the five-form kinetic term gives the following four-dimensional action

$$
\begin{aligned}
& -\frac{1}{4} \int\left\{F^{\Lambda} \star F^{\Sigma}\left(\mathcal{I}+\mathcal{R} \mathcal{I}^{-1} \mathcal{R}\right)_{\Lambda \Sigma}+2 G_{\Lambda} \star F^{\Sigma}\left(\mathcal{R} \mathcal{I}^{-1}\right)_{\Sigma}{ }^{\Lambda}-G_{\Lambda} \star G_{\Sigma} \mathcal{I}^{-1 \Lambda \Sigma}+\right. \\
& \left.-4\left[F^{\Lambda}\left(\mathcal{I}^{-1} \mathcal{R}\right)^{\Sigma}{ }_{\Lambda}+G_{\Lambda} \mathcal{I}^{-1 \Lambda \Sigma}\right] B^{i} e_{\Sigma}^{j} \epsilon_{i j}\right\} .
\end{aligned}
$$

Although the starting action was formulated such as to produce the correct ten-dimensional equations of motion, including the self-duality constraint on the five-form, to compactify the theory we had to set the auxiliary scalar $a(x)$ to zero. Unfortunately, this means that in doing so we lost the self-duality property. To restore it, we add to the above action the Lagrange multiplier

$$
\frac{1}{2} G_{\Lambda} F^{\Lambda}
$$

Integrating out $G_{\Lambda}$ one produces the constraint restoring self-duality (see also 《4)

$$
G_{\Lambda}=\mathcal{I}_{\Lambda \Sigma} \star F^{\Sigma}+\mathcal{R}_{\Lambda \Sigma} F^{\Sigma}+2 B^{i} e_{\Lambda}^{j} \epsilon_{i j}
$$

and the kinetic five-form term reduces to

$$
\frac{1}{2}\left(\mathcal{R}_{\Lambda \Sigma} F^{\Lambda} F^{\Sigma}+\mathcal{I}_{\Lambda \Sigma} F^{\Lambda} \star F^{\Sigma}\right)+B^{i} e_{\Lambda}^{j} \epsilon_{i j} F^{\Lambda}
$$

The first part is just the kinetic term of the vector fields of four-dimensional gauged supergravity [12], whereas the last term reduces to a change of normalization of the reduction of the Wess-Zumino ten-dimensional term, which, after integrating by parts, we can write as

$$
2 \int H^{i} e_{\Lambda}^{j} \epsilon_{i j} A^{\Lambda}
$$

As one can understand the three-form field strength in four dimensions will be dualized to a scalar field strength and therefore the above coupling determines the scalars which are charged under the symmetries gauged by $A^{\Lambda}$.

In a similar fashion, one can compute the kinetic terms and the couplings for the scalar fields coming from the $H^{2}$ and $H^{0}$ sectors. In doing this we use the simplificating assumption that for our Calabi-Yau manifold $h_{(1,1)}=1$ and therefore in the $H^{2}$ sector all the forms are proportional to the Kähler structure $J$. This fixes also the duality relation of any $Y \in h_{(1,1)}$, which is given by

$$
\star Y=\frac{3}{2} \frac{\int Y \wedge J \wedge J}{\int J \wedge J \wedge J}-Y \wedge J .
$$


The five-form $F_{5}$ and the three-forms $H^{i}$ will give rise to three scalar degrees of freedom when expanded in the $H^{2}$ sector:

$$
\begin{aligned}
H^{i} & =d a^{i} Y, \\
F_{5} & =d c Y \wedge Y+f_{3} Y+2\left(a^{i} H^{j}+B^{i} d a^{j}\right) \epsilon_{i j} Y+2 a^{i} d a^{j} \epsilon_{i j} Y \wedge Y .
\end{aligned}
$$

Once again, to implement the self-duality of the five-form in the resulting theory, one has to add to the standard action a multiplier between $f_{3}$ and $d \mathcal{C} \equiv d c+2 a^{i} d a^{j} \epsilon_{i j}$. The integration of $f_{3}$ will leave with the kinetic terms for the physical $c$ and $a^{i}$ fields.

The fourth scalar in this sector comes from the Calabi-Yau metric $\mathrm{i} g_{i \bar{\jmath}}=e^{\sigma} Y_{i \bar{\jmath}}$ and its kinetic term comes from the ten-dimensional Einstein term.

The four scalars of the universal hypermultiplet come instead from the $H^{0}$ sector. For instance from the ten-dimensional one-forms we have:

$$
2 \int \bar{F}_{1} \star F_{1}=2 \int \frac{d \bar{\eta} \star d \eta}{(\eta+\bar{\eta})^{2}}=\int \frac{1}{2 \alpha^{2}}(d \alpha \star d \alpha+d \beta \star d \beta) .
$$

The other two come from the ten-dimensional three-forms properly dualized. To perform this dualization one has to add further Lagrange multipliers $b^{i}$ through

$$
-H^{i} d b^{j} \epsilon_{i j} .
$$

To obtain the effective four-dimensional theory, we still have to remember that the integration over the internal manifold gives a factor of $e^{3 \sigma}$ in front of the four-dimensional Einstein term. Therefore, to go back to the Einstein frame and to normalize the Einstein kinetic term as in [12], we have to reabsorb it by a proper Weyl rescaling of the fourdimensional metric $g_{\mu \nu} \rightarrow \frac{1}{2} e^{-3 \sigma} g_{\mu \nu}$.

Defining the covariant derivative $D b^{i}=d b^{i}+2 e_{\Sigma}^{i} A^{\Sigma}$ and, for ease of notation] $D \mathcal{B}^{1} \equiv$ $D b^{1}-24 a^{1}\left(d \mathcal{C}-2 a^{1} d a^{2}\right), D \mathcal{B}^{2} \equiv D b^{2}-24 a^{2}\left(d \mathcal{C}-4 a^{1} d a^{2}\right)$, one obtains as final action for the four-dimensional scalars:

$$
\begin{aligned}
S_{\text {scal }} & =\int_{4 d}\left[\frac{1}{4 \alpha^{2}}(d \alpha \star d \alpha+d \beta \star d \beta)+3 e^{-4 \sigma} d \mathcal{C} \star d \mathcal{C}+3 d \sigma \star d \sigma+\right. \\
& +\frac{1}{16 \alpha} e^{-6 \sigma}\left(D \mathcal{B}^{1}-\mathrm{i} \bar{\eta} D \mathcal{B}^{2}\right) \star\left(D \mathcal{B}^{1}+\mathrm{i} \eta D \mathcal{B}^{2}\right)+ \\
& \left.+\frac{3}{\alpha} e^{-2 \sigma}\left(d a^{1}-\mathrm{i} \bar{\eta} d a^{2}\right) \star\left(d a^{1}+\mathrm{i} \eta d a^{2}\right)\right] .
\end{aligned}
$$

It is known that these scalars should parametrize a dual quaternionic manifold. To identify the underlying quaternionic structure we now propose some identifications between the above scalars and the usual variables used to parametrize these manifolds [11].

\footnotetext{
${ }^{1}$ The asymmetry between $\mathcal{B}^{1}$ and $\mathcal{B}^{2}$ is due to the integration by parts of the terms of the form $B^{i} d a^{j} a^{k} d a^{l} \epsilon_{i j} \epsilon_{k l}$.
} 
This will become useful for the comparison with the gauged supergravity results, where the new coordinates are more natural.

Although in this computation we have chosen to limit ourselves to the analysis of the scalars describing the hyper sector of the moduli space of a Calabi-Yau with $h_{(1,1)}=1$, which is the minimal set one can consider, we expect that the result will hold also in the generic case, provided one modifies the change of coordinates.

As follows from the the following identifications, the quaternionic manifold described by the above scalars is the coset $G_{2,2} / S O(4)$ [17]

$$
\begin{aligned}
z & =4 a^{2}-2 \mathrm{i} \frac{e^{\sigma}}{\sqrt{\alpha}} \\
S & =e^{3 \sigma} \sqrt{\alpha}-\frac{\mathrm{i}}{2} b^{1}-\frac{1}{4}(C+\bar{C}) \mathcal{R}^{-1}(C+\bar{C}), \\
C_{0} & =-\beta e^{3 \sigma} \alpha^{-3 / 2}-\operatorname{Re} z \operatorname{Re} C_{1}+\frac{\mathrm{i}}{2} b^{2}, \\
C_{1} & =3 \frac{e^{\sigma}}{\sqrt{\alpha}} a^{1}+3 \mathrm{i}\left(c-2 a^{1} a^{2}\right), \\
K & =-\log \left(-\frac{\mathrm{i}}{2}(z-\bar{z})^{3}\right) \\
\tilde{K} & =-\log \left(S+\bar{S}+\frac{1}{2}(C+\bar{C}) \mathcal{R}^{-1}(C+\bar{C})\right),
\end{aligned}
$$

where

$$
\mathcal{N}=\frac{\mathrm{i}}{8}\left(\begin{array}{cc}
-\frac{1}{4}\left(4 z^{3}+12 z^{2} \bar{z}-3 z \bar{z}^{2}+6 \bar{z}^{3}\right) & 3\left(z^{2}+z \bar{z}\right) \\
3\left(z^{2}+z \bar{z}\right) & -9 z-3 \bar{z}
\end{array}\right),
$$

and $\mathcal{R}=(\mathcal{N}+\overline{\mathcal{N}}) / 2$. This implies that one can write the kinetic terms for the above scalars as

$$
\int d^{4} x \sqrt{-g} h_{u v} D_{\mu} q^{u} D^{\mu} q^{v}=\int(u \star \bar{u}+v \star \bar{v}+e \star \bar{e}+E \star \bar{E}),
$$

where [11]

$$
\begin{aligned}
u & =2 e^{(\tilde{K}+K) / 2} Z\left(d C-\frac{1}{2} d \mathcal{N} R^{-1}(C+\bar{C})\right) \\
v & =e^{\tilde{K}}\left(d S+(C+\bar{C}) R^{-1} d C-\frac{1}{4}(C+\bar{C}) R^{-1} d \mathcal{N} R^{-1}(C+\bar{C})\right) \\
e & =P d Z \\
E & =e^{(\tilde{K}-K) / 2} P N^{-1}\left(d C-\frac{1}{2} d \mathcal{N} R^{-1}(C+\bar{C})\right)
\end{aligned}
$$

are the general complex vielbeins, and in our case

$$
\begin{aligned}
Z & =\{1, z\}, \\
P & =\left\{-\sqrt{3} \frac{z}{z-\bar{z}}, \frac{\sqrt{3}}{z-\bar{z}}\right\} \\
N & =\frac{1}{4}\left(\begin{array}{cc}
2 \mathrm{i}\left(z^{3}-\bar{z}^{3}\right) & -3 \mathrm{i}\left(z^{2}-\bar{z}^{2}\right) \\
-3 \mathrm{i}\left(z^{2}-\bar{z}^{2}\right) & 6 \mathrm{i}(z-\bar{z})
\end{array}\right)
\end{aligned}
$$


and the other quantities have been defined above.

We also see now that $b^{1}$ and $b^{2}$ correspond to $-\operatorname{Im} S$ and $\operatorname{Im} C_{0}$ respectively and the introduction of fluxes is producing a gauging of the Peccei-Quinn isometries $S \rightarrow S+\mathrm{i} a$ and $C_{0} \rightarrow C_{0}+\mathrm{i} a$.

We are now in position to express the potential of the theory in terms of this variables. Although this will make the expression of the potential rather complicated, it will be useful in the comparison with the results coming from the four-dimensional gauged supergravity.

The potential is given by the term we obtained from the three-forms (2.14) rescaled with the same rescaling we used for the scalar fields. The ten-dimensional dilaton can be written as

$$
\eta=4 e^{(K-\tilde{K}) / 2}+\mathrm{i}\left[(C+\bar{C}) R^{-1}\right]^{0}
$$

and the rescaling

$$
\frac{e^{-6 \sigma}}{4}=4 e^{(3 \tilde{K}-K) / 2}
$$

therefore the final expression is

$$
\begin{aligned}
\mathcal{V} & =4\left(\bar{L}^{\Lambda} L^{\Sigma}+U^{\Lambda \Sigma}\right)\left[e^{2 \tilde{K}} e_{\Lambda}^{1} e_{\Sigma}^{1}+e^{2 \tilde{K}}\left(16 e^{K-\tilde{K}}+\left(\left[(C+\bar{C}) R^{-1}\right]^{0}\right)^{2}\right) e_{\Lambda}^{2} e_{\Sigma}^{2}\right. \\
& \left.-\left[(C+\bar{C}) R^{-1}\right]^{0} e^{2 \tilde{K}}\left(e_{\Lambda}^{1} e_{\Sigma}^{2}+e_{\Lambda}^{2} e_{\Sigma}^{1}\right)\right] .
\end{aligned}
$$

\section{The potential from gauged supergravity}

Let us now compare this potential with the one that would be expected from the gauging.

The Killing vectors corresponding to the shift symmetries in $\operatorname{Im} S$ and $\operatorname{Im} C_{0}$

$$
k_{\Lambda}=-2 e_{\Lambda}^{2}\left(\begin{array}{c}
i \\
-i \\
0 \\
0
\end{array}\right)+2 e_{\Lambda}^{1}\left(\begin{array}{c}
0 \\
0 \\
i \\
-i
\end{array}\right)
$$

From these vectors one could compute the quaternionic prepotentials $P_{\Lambda}^{x}$ from their standard definition

$$
i_{\Lambda} \Omega^{x}=\nabla P_{\Lambda}^{x}
$$

where $\Omega^{x}$ is the $S U(2)$ curvature of the quaternionic manifold and $\nabla$ is the $S U(2)$ covariant derivative. In our case these vectors correspond to Peccei-Quinn symmetries under which the Lie derivative of the $S U(2)$ connection vanishes and therefore the above expression can be simplified [6]. The prepotentials can be given by the contraction of this Killing vectors with the $S U(2)$ connection:

$$
\mathcal{P}_{\Lambda}^{x}=\omega_{u}^{x} k_{\Lambda}^{u}
$$


Since the potential which comes from gauging abelian isometries of the quaternionic manifold can be written as [12]:

$$
\mathcal{V}=\left(U^{\Lambda \Sigma}-3 \bar{L}^{\Lambda} L^{\Sigma}\right) P_{\Lambda}^{x} P_{\Sigma}^{x}+4 \bar{L}^{\Lambda} L^{\Sigma} h_{u v} k_{\Lambda}^{u} k_{\Sigma}^{v}
$$

or, in terms of the prepotentials, as

$$
\mathcal{V}=\left(U^{\Lambda \Sigma}-3 \bar{L}^{\Lambda} L^{\Sigma}\right) P_{\Lambda}^{x} P_{\Sigma}^{x}+\frac{1}{3} \bar{L}^{\Lambda} L^{\Sigma} h^{u v} \nabla_{u} P_{\Lambda}^{x} \nabla_{v} P_{\Sigma}^{x}
$$

we just need the explicit expression of the connection to obtain it.

The quaternionic vielbeine which give the scalar action (2.34) are

$$
\mathcal{U}^{\alpha A}=\frac{1}{\sqrt{2}}\left(\begin{array}{cccc}
u & e & -\bar{v} & -\bar{E} \\
v & E & \bar{u} & -\bar{e}
\end{array}\right) .
$$

From these we can define as usual the metric and the curvature

$$
\begin{aligned}
h_{u v} & =\mathcal{U}_{u}^{\alpha A} \mathcal{U}_{v}^{\beta B} C_{\alpha \beta} \epsilon_{A B} \\
\Omega^{x} & =\mathrm{i} C_{\alpha \beta}\left(\sigma^{x}\right)_{A}^{C} \epsilon_{C B} \mathcal{U}^{\alpha A} \wedge \mathcal{U}^{\beta B}=d \omega^{x}+\frac{1}{2} \epsilon^{x y z} \omega^{y} \omega^{z}
\end{aligned}
$$

and from this latter, we can derive the connection, which is given by

$$
\begin{aligned}
\omega^{1} & =\mathrm{i}(u-\bar{u}), \\
\omega^{2} & =(u+\bar{u}), \\
\omega^{3} & =\frac{\mathrm{i}}{2}(\bar{v}-v)+\frac{\mathrm{i}}{2} \frac{\bar{Z} N d Z-Z N d \bar{Z}}{\bar{Z} N Z} .
\end{aligned}
$$

Contracting now with the Killing vectors (3.1) we obtain the prepotentials

$$
\mathcal{P}_{\Lambda}=\left(\begin{array}{c}
8 e^{(\tilde{K}+K) / 2} e_{\Lambda}^{2} \\
0 \\
2 e^{\tilde{K}} e_{\Lambda}^{1}-2 e^{\tilde{K}}\left[(C+\bar{C}) R^{-1}\right]^{0} e_{\Lambda}^{2}
\end{array}\right)
$$

For the case under consideration such prepotentials satisfy the non-trivial relation

$$
P_{\Lambda}^{x} P_{\Sigma}^{x}=h_{u v} k_{\Lambda}^{u} k_{\Sigma}^{v}
$$

This implies that the final structure of the potential goes through a drastic simplification and is given by

$$
\mathcal{V}=\left(U^{\Lambda \Sigma}+\bar{L}^{\Lambda} L^{\Sigma}\right)\left(P_{\Lambda}^{x} P_{\Sigma}^{x}\right)
$$

By a direct evaluation one can see that it is the same one as that presented in (2.44).

This result differs from the one presented in [6], due to the relation (3.13) that now is satisfied. This happens because the truncation performed in [6 looses a contribution to the square of the Killing vectors $h_{u v} k^{u} k^{v}$ which comes from the $E$ complex vielbein which was discarded. If one instead first considers the full metric (which contains also 
the $E \otimes \bar{E}$ term) and then truncates to the subsector described by $C_{1}=0$, the result agrees with the one we presented. Indeed, the above-mentioned term gives an additional contribution equal to

$$
e^{\tilde{K}-K}\left[P N^{-1}\right]^{0}\left[\bar{P} \bar{N}^{-1}\right]^{0}
$$

Using the general property

$$
P \cdot P^{\dagger}=-\frac{1}{\bar{Z} N Z}\left(N-\frac{(N Z)(\bar{Z} N)}{\bar{Z} N Z}\right),
$$

and the equality

$$
N^{00}=-\frac{2}{\bar{Z} N Z}
$$

which holds for the $G_{2(2)} / S O(4)$ coset, one obtains that 3.15 gives the additional contribution which is essential to make the equation (3.13) satisfied.

\section{Comments}

Inspecting the equation defining the potential

$$
\mathcal{V}=\left(U^{\Lambda \Sigma}+\bar{L}^{\Lambda} L^{\Sigma}\right)\left(P_{\Lambda}^{x} P_{\Sigma}^{x}\right)
$$

one can see that it is positive definite, i.e. $\mathcal{V} \geq 0$ and that it vanishes if and only if $\left(P_{\Lambda}^{x} P_{\Sigma}^{x}\right)=0$.

It is easy to show that the requirement of stationary points under variation of the hypermultiplets implies that all the coupling constants be zero. The potential has indeed a run-away behaviour in this sector as can be understood by expressing the above potential in terms of the four-dimensional dilaton $\phi_{4} \sim e^{\tilde{K}}$. Also, going back to the original ten-dimensional variables, one sees that this same potential is a function of the tendimensional dilaton/axion $\eta$ and the inverse volume of the Calabi-Yau $e^{-6 \sigma}$. Variation under $\sigma$ shows a critical point for the decompactification limit $\sigma \rightarrow \infty$.

An interesting feature of this potential is that it can be written in a form which is very close to that of a pure $\mathcal{N}=1$ supergravity theory. If we use the " $\mathcal{N}=1$ section"

$$
L=e^{\frac{1}{2} \mathcal{K}} W
$$

where we defined the superpotential [9, 10]

$$
W=e^{-\mathcal{K} / 2} L^{\Lambda}\left(P_{\Lambda}^{3} \pm \mathrm{i} P_{\Lambda}^{1}\right), \quad \mathcal{K}=K_{V}-(K+\tilde{K}),
$$

the potential becomes

$$
\mathcal{V}=-3 \bar{L} L+g^{i \bar{\jmath}} \nabla_{i} L \nabla_{\bar{\jmath}} \bar{L}+h^{u v} \nabla_{u} L \nabla_{v} \bar{L}
$$


with

$$
\nabla_{A} \equiv \partial_{A}+\frac{1}{2} \partial_{A} \mathcal{K}
$$

Although the form of (4.4) looks like that of a pure $\mathcal{N}=1$ potential, it is not yet so, since the quaternionic metric $h_{u v}$ cannot in general be derived from the Kähler potentials $K+\tilde{K}$. It has been shown [11] that the necessary condition for such metric to be also Kähler is given by the holomorphicity of the matrix $\mathcal{N}_{\Lambda \Sigma}$. This restricts the possible quaternionic manifolds to be $S U(2, n+1) / S U(2) \times S U(n+1) \times U(1)$, which includes the truncation to the Universal hypermultiplet alone. Unfortunately, as one can argue from the above example, these are not the manifolds which are chosen by the Calabi-Yau compactifications. As for such manifolds the equation (3.17) does not hold anymore, the quaternionic prepotentials will not satisfy (3.13) and this prevents us from the possibility of writing the potential in the (4.1) form and as a further consequence as (4.4).

The only chance to make of the (4.4) potential a real $\mathcal{N}=1$ potential is to truncate the theory to the degrees of freedom given by the ten-dimensional dilaton/axion scalars $\eta$, the volume $\sigma$ and its axionic partner $c$. This cannot be achieved by just fixing their value to a constant in the $\mathcal{N}=2$ theory, as the obtained potential has no critical points at all. It could anyway be that there is a consistent truncation of such theory to an $\mathcal{N}=1$ one, where the above scalars become chiral multiplets. In performing this truncation, the form of the $\mathcal{N}=1$ superpotential $W$ must be consistent with the reduction of the supersymmetry laws and this fixes it to be of the form given in (4.3).

The final theory will be then a real $\mathcal{N}=1$ supergravity theory, whose potential form (4.4) (with $h_{u v}$ now a Kähler metric) would then be justified.

A further consistency problem is given by the fact that $W$ is holomorphic in the coordinates of the vector scalar manifold, but it is not in general in terms of the hypers?.

One can also understand that the theory we are describing is a genuine $\mathcal{N}=2$ theory analyzing the supersymmetry laws of the various Fermi fields. The shifts of the gravitini, gaugini and hyperini are given by

$$
S_{A B}=-\frac{\mathrm{i}}{2}\left(\begin{array}{cc}
-P^{1} & P^{3} \\
P^{3} & P^{1}
\end{array}\right)_{A B},
$$

where now $P^{x} \equiv L^{\Lambda} P_{\Lambda}^{x}$

$$
W^{i A B}=\mathrm{i} g^{i \bar{\jmath}}\left(\begin{array}{cc}
-\nabla_{\bar{\jmath}} \bar{P}^{1} & \nabla_{\bar{\jmath}} \bar{P}^{3} \\
\nabla_{\bar{\jmath}} \bar{P}^{3} & \nabla_{\bar{\jmath}} \bar{P}^{1}
\end{array}\right)^{A B}
$$

\footnotetext{
${ }^{2}$ For the general conditions under which such a truncation is consistent we refer the reader to 18,19 . Related aspects were also presented in J. Louis' seminar at Humboldt Uni. in May 2001.

${ }^{3}$ We thank R. D'Auria and S. Ferrara for discussions on this point.
} 
and

$$
\mathcal{N}^{\alpha A}=\frac{\mathrm{i}}{\sqrt{2}}\left(\begin{array}{cc}
-\bar{P}^{1} & \bar{P}^{3} \\
0 & i_{\bar{k}} E \\
\bar{P}^{3} & \bar{P}^{1} \\
-i_{\bar{k}} \bar{E} & 0
\end{array}\right)^{\alpha A}
$$

From the gravitini transformation law, one notice that if there is a vacuum such that

$$
P^{1}= \pm \mathrm{i} P^{3} \quad \Leftrightarrow \quad W=0
$$

the matrix (4.6) becomes degenerate and therefore one can preserve half supersymmetry.

Due to the reality properties of the quaternionic prepotentials, this same condition (4.9) implies that the $L^{\Lambda}$ sections cannot be chosen to be all independent. This, as a further consequence, implies that there is no holomorphic prepotential for the Kähler potential of the vector scalar manifold [11].

At the same time, one can see that the gaugini transformation law implies that also the derivatives must satisfy an analogous relation

$$
\nabla_{i} P^{1}= \pm \mathrm{i} \nabla_{i} P^{3} \quad \Leftrightarrow \quad \nabla_{i} W=0
$$

This is still possible to satisfy again by a choice of section which is not linearly independent [2, 3].

Then there are the hyperini transformations. As one can see, the same condition (4.9) applied to the $\mathcal{N}^{\alpha A}$ matrix implies that half of the hyperini supersymmetry laws can vanish, but still the other half cannot. To preserve $\mathcal{N}=1$ one should further require that $i_{k} E=0$, which is a much stronger constraint and in our case this has solutions only in the limit $e^{\tilde{K}-K}=0$.

At the same time, these considerations let us conclude that there is still the chance to find $\mathcal{N}=1$ vacua in the decoupling limit where the hypermultiplets freeze. In this limit one would end with $\mathcal{N}=1$ supersymmetry preserved in the visible sector and $\mathcal{N}=0$ in the hidden one [3].

We conclude this brief analysis by recalling that the final form of the potential still respects the $S L(2, \mathbb{Z})$ invariance of the ten-dimensional action. Since the reduction of the ten-dimensional dilaton/axion field to four dimensions is not straightforward, the action of such symmetry on the four-dimensional fields is non-trivial [5]. Anyway, we saw that the form of the potential one derives by direct compactification depends only on the ten-dimensional field $\eta$ and the rescaling needed to go to the Einstein frame. Moreover, before this rescaling the potential was $S L(2, \mathbb{Z})$ invariant, once the appropriate action on the charges was chosen (they must transform as vectors). Since the quantity by which we rescaled it is proportional to the Calabi-Yau volume is also $S L(2, \mathbb{Z})$ invariant the final potential preserves this symmetry. 


\section{Acknowledgments.}

I am glad to thank R. D'Auria and S. Ferrara for the many enlightening discussions and comments and for informing me on the related work in progress [18]. I also would like to thank J. Louis for informing me of related work in progress [19] and A. Klemm and D. Lüst for discussions. This research is supported by the EC under RTN project HPRN-CT-2000-00131.

\section{References}

[1] I. Antoniadis, H. Partouche, and T. R. Taylor, "Spontaneous breaking of N=2 global supersymmetry," Phys. Lett. B372 (1996) 83-87, hep-th/9512006.

[2] S. Ferrara, L. Girardello, and M. Porrati, "Minimal higgs branch for the breaking of half of the supersymmetries in N=2 supergravity," Phys. Lett. B366 (1996) 155-159, hep-th/9510074.

[3] S. Ferrara, L. Girardello, and M. Porrati, "Spontaneous breaking of $\mathrm{N}=2$ to $\mathrm{N}=1$ in rigid and local supersymmetric theories," Phys. Lett. B376 (1996) 275-281, hep-th/9512180.

[4] A. Ceresole, R. D'Auria, and S. Ferrara, "The symplectic structure of N=2 supergravity and its central extension," Nucl. Phys. Proc. Suppl. 46 (1996) 67-74, hep-th/9509160.

[5] R. Bohm, H. Gunther, C. Herrmann, and J. Louis, "Compactification of type IIb string theory on calabi-yau threefolds," Nucl. Phys. B569 (2000) 229-246, hep-th/9908007.

[6] J. Michelson, "Compactifications of type iib strings to four dimensions with non-trivial classical potential," Nucl. Phys. B495 (1997) 127-148, hep-th/9610151.

[7] T. R. Taylor and C. Vafa, "RR flux on Calabi-Yau and partial supersymmetry breaking," Phys. Lett. B474 (2000) 130-137, hep-th/9912152.

[8] S. Gukov, "Solitons, superpotentials and calibrations," Nucl. Phys. B 574, 169 (2000) hep-th/9911011.

[9] P. Mayr, "On supersymmetry breaking in string theory and its realization in brane worlds," Nucl. Phys. B 593, 99 (2001) hep-th/0003198. 
[10] G. Curio, A. Klemm, D. Lust, and S. Theisen, "On the vacuum structure of type II string compactifications on Calabi-Yau spaces with H-fluxes," hep-th/0012213.

[11] S. Ferrara and S. Sabharwal, "Quaternionic manifolds for type II superstring vacua of calabi-yau spaces," Nucl. Phys. B332 (1990) 317.

[12] L. Andrianopoli et al., " $\mathrm{N}=2$ supergravity and $\mathrm{N}=2$ super Yang-Mills theory on general scalar manifolds: Symplectic covariance, gaugings and the momentum map," J. Geom. Phys. 23 (1997) 111-189, hep-th/9605032.

[13] G. Dall'Agata, K. Lechner and D. Sorokin, "Covariant actions for the bosonic sector of D = 10 IIB supergravity," Class. Quant. Grav. 14, L195 (1997) hep-th/9707044.

[14] G. Dall'Agata, K. Lechner, and M. Tonin, " $\mathrm{D}=10, \mathrm{~N}=\mathrm{IIb}$ supergravity: Lorentz-invariant actions and duality," JHEP 07 (1998) 017, hep-th/9806140.

[15] P. Pasti, D. Sorokin, and M. Tonin, "On Lorentz invariant actions for chiral p-forms," Phys. Rev. D55 (1997) 6292-6298, hep-th/9611100.

[16] H. Suzuki, "Calabi-Yau compactification of type IIb string and a mass formula of the extreme black holes," Mod. Phys. Lett. A11 (1996) 623-630, hep-th/9508001.

[17] M. Bodner and A. C. Cadavid, "Dimensional reduction of type IIb supergravity and exceptional quaternionic manifolds," Class. Quant. Grav. 7 (1990) 829.

[18] L. Andrianopoli, R. D’Auria, and S. Ferrara, "work in progress".

[19] J. Louis, " work in progress". 